\title{
HispanismeS
}

Revue de la Société des Hispanistes Français

$17 \mid 2021$

Murs, barrières, obstacles dans les mondes

hispaniques I

\section{El valor de la lengua española hoy}

La valeur de la langue espagnole aujourd'hui

The Value of the Spanish Language today

\section{Darío Villanueva}

\section{OpenEdition}

\section{Journals}

\section{Edición electrónica}

URL: https://journals.openedition.org/hispanismes/14956

DOI: 10.4000/hispanismes. 14956

ISSN: 2270-0765

Editor

Société des Hispanistes Français

\section{Referencia electrónica}

Darío Villanueva, «El valor de la lengua española hoy», HispanismeS [En línea], 17 | 2021, Publicado el

01 junio 2021, consultado el 27 enero 2022. URL: http://journals.openedition.org/hispanismes/14956 ; DOI: https://doi.org/10.4000/hispanismes.14956

Este documento fue generado automáticamente el 27 enero 2022.

\section{(c) $(1) \Theta \Theta$}

Les contenus de cette revue sont mis à disposition selon les termes de la Licence Creative Commons Attribution - Pas d'Utilisation Commerciale - Pas de Modification 4.0 International. 


\title{
El valor de la lengua española hoy
}

\author{
La valeur de la langue espagnole aujourd'hui \\ The Value of the Spanish Language today
}

Darío Villanueva

1 Antes de nada, y no por mero prurito académico, me siento obligado a determinar el significado exacto que le quiero dar a la palabra valor que actúa como emblema en el propio título de esta conferencia que he escrito para inaugurar el decimoquinto coloquio de la Asociación de Hispanistas, ALMOREAL, acrónimo de las Universidades de Angers, Le Mans y Orléans, que comparten el objetivo común de prestar atención preferente a las relaciones entre Europa y Latinoamérica.

2 Para ello, recurriré, lógicamente, a la vigesimotercera edición del Diccionario de la Lengua Española que en su versión digital, actualizada desde mi época de director al final de cada año, recibe mensualmente una media de sesenta millones de consultas.

Las dos acepciones iniciales del lema valor son: primera, «Grado de utilidad o aptitud de las cosas para satisfacer las necesidades o proporcionar bienestar o deleite»; y segunda, «Cualidad de las cosas, en virtud de la cual se da por poseerlas cierta suma de dinero o equivalente».

4 Pues bien, de un tiempo a esta parte se ha prestado novedosa y significativa atención al valor de nuestro idioma de acuerdo con la segunda de las acepciones mencionadas, la estrictamente económica.

5 Fue pionero en este planteamiento el académico y vicedirector de la RAE don Ángel Martín Municio, bajo cuya iniciativa y dirección se publicó en 2003 una primera obra titulada precisamente El valor económico de la lengua española que recogía el resultado de la investigación, financiada por uno de los grandes bancos españoles (Santander), en la que intervinieron siete economistas.

6 Lo que Martín Municio les encomendó a sus colaboradores, y no llegó a ver publicado por su fallecimiento en 2002, fue determinar el valor económico de la lengua española mediante la cuantificación de su porcentaje en el producto interior bruto (PIB) del país. Para ello, los autores se ocuparon de medir la aportación a nuestra economía de todas las actividades para cuyo desarrollo es imprescindible una presencia determinante del 
idioma. No se circunscribieron a la valoración de las llamadas "industrias de la lengua", esto es, la lengua como producto, sino que ampliaron su campo de atención a todas las producciones en las que el idioma constituye un elemento esencial.

7 La selección de actividades y productos vinculados a la utilización de la lengua incluía cuatro grandes apartados. El primero resulta obligado: todas las actuaciones generadoras de bienes y servicios relacionados directamente con el idioma. Se trata, pues, de la industria editorial, de la producción de objetos para la escritura, de las citadas "industrias de la lengua", de la enseñanza en sus distintas variedades, de la publicidad y de la administración pública. El segundo apartado correspondería a los abastecedores de materias primas y otros insumos esenciales para las actividades anteriormente señaladas. En tercer lugar, aparecen las acciones de comercialización y distribución auxiliares de los dos grupos anteriores. Y finalmente, un cuarto apartado dedicado al resto de sectores activos relacionados con la cultura, como pueden ser la edición de otros elementos no literarios, por ejemplo musicales, y la órbita de los medios de comunicación no incluidos con anterioridad: televisión, radio, cine, etc.

Este estudio de índole macroeconómica se centra en el decenio anterior al año de su publicación (2003), y extrapola sus resultados hasta 2004. La metodología aplicada estrictamente a la realidad económica española de la época podría, sin embargo, aplicarse a la valoración de nuestra lengua común en cualquier otro país hispánico.

Los resultados obtenidos para los años antes reseñados indican que la valoración de nuestra lengua en precios corrientes alcanza en 2001 un porcentaje del PIB de un 15,0\%, ratio que se mantendría durante el trienio 2002-2004.

Posteriormente, una importante multinacional española (Telefónica) promovió y financió entre 2006 y 2014 una exhaustiva investigación bajo el título de Valor económico del español codirigida por el catedrático universitario y economista José Luis García Delgado junto con José Antonio Alonso y Juan Carlos Jiménez. Fruto de este enorme esfuerzo fue la publicación de catorce monografías en forma de libro, entre las cuales figura ya la que mi compañero de la RAE y la USC Guillermo Rojo, secundado por la lexicógrafa de la Academia Mercedes Sánchez, dedicó a una nueva dimensión que no estaba todavía en el horizonte de Martín Municio y su equipo: El español en la red.

Utilizando como en la iniciativa anterior una metodología macroeconómica, los investigadores codirigidos por José Luis García Delgado llegaron a conclusiones actualizadas no muy diferentes a las de 2003. Fundamentalmente, que la lengua española aportaba aproximadamente el 16 por ciento del valor del PIB (164.000 millones de euros de 2014) y otro tanto del empleo en España (3.5 millones de puestos de trabajo), porcentajes similares a los que presumiblemente se encontrarían en las economías mayores de la América hispana de serles aplicado el mismo patrón de trabajo. Por otra parte, se estimaba que las industrias culturales, tan vinculadas al idioma, supondrían el 3 por ciento de estas economías.

Junto a estas conclusiones, relativas al peso del español en términos de renta y empleo, esta investigación, resumida recientemente en sus términos fundamentales por José Luis García Delgado en el volumen colectivo Más de 555 millones podemos leer este libro sin traducción, analizó asimismo en qué medida nuestra lengua actúa como palanca generadora de efectos multiplicadores de intercambios comerciales y flujos de inversión. Se llega, así, a la conclusión de que el español cuadriplica los intercambios y transacciones entre los países en los que se habla, y de que el hecho de compartir esta misma lengua septuplica los flujos bilaterales de inversión directa exterior. No es difícil 
concordar, pues, con García Delgado en que una lengua común como la nuestra actúa como un impagable instrumento de internacionalización -por no decir globalizaciónempresarial, pues posibilita considerables ahorros en cuanto a los llamados "costes de transacción", que algunas empresas multinacionales llegan a fijar en cerca del 2 por ciento de su total de ingresos.

Resulta también interesante otra consideración. El conocimiento del español como lengua materna por parte de los inmigrantes latinoamericanos representa un premio salarial de hasta un 30 por ciento en el mercado de nuestro país, amén de facilitar obviamente una más fácil integración laboral y social. Y se apunta a la vez acertadamente que en los Estados Unidos ese premio se está dando también siempre en clave de un bilingüismo inglés/español cada vez más valorado por los empleadores.

Pero en esta mi presentación no puedo olvidarme del primer significado de valor como «Grado de utilidad o aptitud de las cosas para satisfacer las necesidades o proporcionar bienestar o deleite». Guiémonos ahora no ya por la economía, sino por beneficios en principio intangibles como satisfacción, bienestar, provecho inmaterial.

The Ethnologue: Languages of the World es una publicación elaborada por un instituto dedicado a documentar estadísticamente la realidad de unas 6.900 lenguas de todo el mundo. Según esta referencia, el español es la segunda lengua del mundo por número de hablantes nativos, 477 millones, solo por detrás del chino mandarín, lo que representa un $7,8 \%$ de la población mundial. Ocupa el mismo lugar en cuanto al número de sus estudiantes no nativos, más de veinte millones, importante rubro en el que hay que destacar el creciente interés por el español en Asia y el África subsahariana. En Costa de Marfil, por caso, hay ya 568.561 estudiantes de nuestra lengua y 2.319 profesores, solo en media y secundaria. No dispongo de cifras actualizadas en lo que se refiere al conjunto del Magreb, región mediterránea en la que el español cuenta con circunstancias favorables de índole geográfica, histórica y cultural, amén de una presencia excepcional de centros del Instituto Cervantes aquí en Argel, en Argelia, en Marruecos y en Túnez. Según fuentes acreditadas, en 2017 se computaban solo en Marruecos 80.000 estudiantes en diversos centros educativos.

16 Por otra parte, el español es la tercera lengua en internet, la segunda en Facebook y Twitter. Y en consonancia con la dimensión económica que ya hemos planteado, según las previsiones de la Insead Business School, en 2030 se convertirá en la segunda de intercambio comercial tras el chino. En cifras totales, somos 572 millones las personas que hoy la utilizamos, y las previsiones para 2050 se sitúan en los 750 millones. No es, por lo tanto, hiperbólico ni chovinista calificar hoy por hoy al español como lengua ecuménica o lengua global, de la que se ocupan 24 Academias pertenecientes a otros tantos países de África, América, Asia y Europa. Es la segunda desde tal consideración después del inglés que, no lo olvidemos, disfruta de un estatuto privilegiado como lingua franca ni más ni menos, déjenme decirlo sin pelos en la lengua, porque la lengua de Shakespeare y Henry Ford ganó la segunda guerra mundial.

18 Este año 2019 se conmemoró (sin pena ni gloria, y bien que me duele decirlo) el quinto centenario del comienzo de la expedición capitaneada por Fernando de Magallanes y concluida tres años más tarde con la llegada a Sanlúcar de Barrameda de la nao Victoria al mando de Juan Sebastián Elcano. Se completaba así la primera circunnavegación del globo terráqueo, el atisbo inicial de lo que hoy se ha dado en llamar, precisamente, globalización. Se trata de la característica más determinante de nuestra época, y de una 
civilización decisivamente marcada por los avances tecnológicos de la sociedad de la comunicación.

Quisiera mencionar un ejemplo práctico de esta globalización que afecta a nuestra lengua común. Hasta ahora, y desde 1780, el Diccionario de la lengua española ha sido un libro que en 2002 se digitalizó para ofrecerlo en acceso libre en la red. Ya en 2020 fueron 1000 millones las consultas que se hicieron desde doscientos países del mundo, no solo desde dispositivos fijos como las computadoras, sino también desde tabletas o teléfonos inteligentes. Nunca, en su historia plurisecular, esta obra ha podido ejercer tanta influencia sobre los hispanohablantes como ahora lo hace.

20 A partir de los datos estadísticos antes apuntados de acuerdo con The Ethnologue yo destacaría la fortaleza del español como lengua nativa, como la lengua materna que se aprende en el ámbito familiar. El crecimiento vegetativo de muchos de los países hispanos asegura una continuidad expansiva que, sin duda, podrá refrenarse a medida que los cambios sociales vayan modificando las tasas de natalidad en aquellos en los que hoy en día aún son muy altas, pero junto a esto me parece fundamental reparar en el crecimiento potencial del español en dos grandes potencias no hispanas, de características muy diferentes la una y la otra, en las que, sin embargo, la evolución de nuestro idioma se muestra muy prometedora. Me refiero a los Estados Unidos y la República Popular China.

21 Además de la robustez demográfica de México, con sus 124 millones de habitantes, los académicos y sociolingüistas estamos fascinados, y a la vez expectantes, acerca de la situación actual del español en los Estados Unidos, y su previsible evolución.

Hay, a este respecto, opiniones diversas, a veces encontradas. Frente a los que proclaman la endeblez de los argumentos estadísticos, sociales, políticos, económicos y culturales esgrimidos, yo me sitúo en el grupo de los que creemos en la firmeza del porvenir hispano de este gran país. Hoy, casi el 18\% de la población en los Estados Unidos es hispana.

23 Allí más de 40 millones de personas hablan español con pleno dominio, y otros 15 poseen una competencia más o menos amplia. Repárese que la tercera lengua, el chino, es hablado tan solo por unos tres millones de norteamericanos. Según un estudio del Pew Research Center, nuestro idioma es el más utilizado en los hogares estadounidenses tanto en la comunidad hispana como en las demás, solo superado por el inglés. Por otra parte, el español es el más estudiado, con ocho millones de alumnos, la mitad de ellos preuniversitarios.

Otros datos encierran no menor interés. La Oficina del Censo certificaba en 2016 que la edad media de nuestra comunidad era la más joven, en torno a los 28 años, muy por debajo de la siguiente, la afroamericana, con 34. Y el Observatorio de la lengua española y las culturas hispánicas en los Estados Unidos del Instituto Cervantes y la Universidad de Harvard afirma que el $76 \%$ de los hispanos domina el español o es bilingüe. Pero incluso me parece más relevante que ahora -y no siempre fue así- el $95 \%$ de esta población considere muy importante que los jóvenes, hispanos o no, hablen español.

La propia Oficina del Censo espera que el crecimiento de nuestra comunidad continúe a un ritmo estable. En el último año, uno de cada dos nacimientos ha sido hispano. En 2050 se calcula que la población de los Estados Unidos será de 398 millones de personas, de las cuales 106 serían hispanas. 
Y no menor importancia tiene el peso político que esta comunidad va cobrando sin parar. En las últimas elecciones de 2016 hubo 27,3 millones de hispanos con derecho a voto, un aumento de un $70 \%$ si se compara con los datos de 2000 . Ello quiere decir que el 11\% del voto nacional fue hispano, mientras que, por ejemplo, en 2004 había sido solo un $4 \%$, tal y como apunta un reciente informe sobre el español en la política de los Estados Unidos elaborado por The Spanish Council.

Mucho se ha avanzado, sin duda, en la presencia de nuestra lengua en la vida política norteamericana desde las elecciones de 1960, las primeras en las que entró el español de la mano de Jackie Kennedy, que pidió el voto en nuestra lengua para su marido. Bien es cierto que con la presidencia de Donald Trump soplaron vientos poco favorables, pero la solidez de las cifras electorales y el creciente empoderamiento de la comunidad hispana inspiran confianza, así como la consideración muy generalizada de que el español es una lengua universal, que transmite además valores firmes y crecientes en los planos económico, social, periodístico y comunicativo, político, cultural, deportivo o científico. Canales de televisión como Univisión, Estrella TV o Telemundo ya compiten en audiencia con las grandes cadenas del país, y se publican periódicos en español en California, Florida, Illinois, Nueva York o Texas.

Los lingüistas diferencian entre dos situaciones distintas en lo que al contacto entre lenguas se refiere: el bilingüismo y la diglosia. Detrás del distingo están las relaciones de poder. Una cosa es la convivencia de dos lenguas en un plano de razonable equidad y otra cuando la lengua A, así denominada por los expertos, representa la riqueza, el poder y el prestigio social, mientras que la lengua B aparece subordinada como perteneciente a quienes también lo están en una determinada sociedad.

Tengo para mí que, aparte de los datos estadísticos, y al margen incluso de un cierto enrarecimiento del clima político desde el comienzo de la presidencia de Donald Trump, el español está afianzando su posición como un idioma en condiciones de servir sin limitación alguna a la sociedad norteamericana en convivencia bilingüe con el inglés. Y ello no es mérito de ninguna Academia, sino de los millones de mujeres y de hombres, niños, jóvenes y mayores, que hacen de una lengua universal como es la nuestra la herramienta de sus trabajos y de sus días, pero también el emblema de su pertenencia a una comunidad extendida por cuatro continentes, acrisolada por una Historia compleja, dueña de una cultura poderosa y diversa, y abierta a un futuro prometedor.

A principios de 2018 el gobierno chino aprobó un nuevo diseño de la enseñanza secundaria y del bachillerato en el que se contempla la inclusión de otras tres lenguas extranjeras en la oferta del currículo educativo además de las tres ya existentes, el inglés, el japonés y el ruso. Se trata ahora del español, el francés y el alemán.

Según informaciones posteriores, nos consta que las autoridades competentes manifestaban la necesidad de contar con apoyo para afrontar los dos grandes retos que su decisión plantea: por una parte, disponer de suficiente profesorado, bien nacional bien de nativos hispanohablantes, y por otra, contar con instrumentos docentes ad hoc: materiales didácticos, unidades y ejercicios prácticos, documentación y bibliografía sobre la lengua española. Estos problemas han lastrado desde un principio las magníficas expectativas que se abrieron a principio de este siglo cuando se promulgó en Brasil otra ley para hacer obligatoria, gradualmente, la oferta del español en enseñanza media, y opcional en los tres últimos cursos de primaria. 
En China cada año se presentan a las pruebas de acceso a la Universidad nueve millones de estudiantes, y las superan ocho. Quiero decir ocho millones (de otro modo estaríamos hablando no tanto de una selección sino de una verdadera masacre selectiva). Sumando los ciclos de secundaria y de bachillerato, son más de sesenta millones los alumnos a los que, en aplicación del nuevo diseño curricular antes mencionado, se les ofrecerá la posibilidad de estudiar español antes de entrar en la Universidad.

En este momento, de las 3000 Universidades que existen en China, unas 120 cuentan con departamentos de español. En aquel total, destacan ochenta catalogadas como "nacionales", algunas de ellas especializadas en los llamados "estudios internacionales", en consonancia con lo que desde hace ya varios decenios constituye una de las prioridades estratégicas del gobierno: la apertura al exterior, la "internacionalización".

Entre las ocho mejores universidades nacionales de este sector se encuentra SISU, la Universidad de Estudios Extranjeros de Shanghai, alma mater de destacados políticos, diplomáticos, profesores y ejecutivos de empresa.

Según informaciones proporcionadas por el Instituto Cervantes, que viene ejerciendo desde comienzos de siglo una labor admirable en sus centros de Pekín y Shanghai, se sitúa en torno a los 50.000 el número de ciudadanos chinos que estudian español. La cifra homóloga correspondiente al inglés es abrumadora (varias decenas de millones), lo que da fe de las posibilidades de crecimiento que nuestro idioma tiene, dado su potencial y su condición de segunda lengua del mundo por el número de hablantes nativos, después, precisamente, del chino mandarín.

Esa expansión será posible en gran medida gracias a las disposiciones del nuevo plan curricular del que partimos, todo ello favorecido por la actitud de atenta curiosidad que la sociedad china manifiesta últimamente hacia las comunidades y las culturas que se expresan en español, y el evidente interés estratégico del gobierno hacia la región de Latinoamérica, lo que no ensombrece su atención a España como cuna del español y miembro de la Unión Europea. Como síntoma de esta consideración puede tomarse la visita de Estado a España del presidente Xi Jinping en noviembre de 2018, que luego siguió camino hacia Buenos Aires.

7 La Real Academia Española, cuando tuvo noticia de estas posibilidades abiertas para el español recurrió enseguida a su académico correspondiente Lu Jinseng, catedrático de SISU y presidente de la Comisión nacional para la enseñanza del español en su país, organismo del ministerio de Educación.

8 El propósito de la RAE era ofrecer un instrumento totalmente novedoso, inexistente en el caso de las otras lenguas antes mencionadas, para proporcionar a los centros de secundaria y bachillerato toda la documentación pertinente sobre la gramática, la ortografía y el léxico del español mediante una plataforma digital interactiva especialmente pensada para la enseñanza de nuestro idioma como lengua extranjera. Su nombre es ENCLAVE RAE.

Huelga decir que, conforme a la política panhispánica que desde decenios ha hecho suya la Asociación de Academias de la Lengua Española ASALE, lo que se enseñará es el español de todo el mundo, sin que ninguna de sus variantes locales tenga primacía sobre las demás. 

ENCLAVE RAE y de ese encuentro surgió la realización de un Simposio internacional de la Enseñanza del español en China que se ha desarrollado los días 19 y 20 de septiembre con asistencia, fundamentalmente, de 130 especialistas y profesores de español en Universidades y Escuelas secundarias. A tal fin, se desplazó a Shanghai una delegación de académicos y técnicos de la RAE, que a lo largo de una semana, además de participar en el simposio, mantuvieron intensos contactos con las autoridades universitarias y los hispanistas chinos, así como con los representantes diplomáticos y comerciales de la Embajada del Reino de España, con los responsables del Instituto Cervantes y con los medios informativos locales y españoles.

Igualmente, se acordaron tres convenios de colaboración entre cuyas estipulaciones se cuenta la creación de un CENTRO CONJUNTO DE INVESTIGACIÓN RAE/SISU. Uno de sus objetivos principales será la promoción de la plataforma digital ENCLAVE RAE como herramienta de apoyo didáctico a la implantación de la enseñanza del español en la secundaria y el bachillerato chino y su adaptación, en lo que fuere necesario, a las peculiaridades de su profesorado y alumnado.

trata, sin duda, de una operación de máxima importancia, basada en una herramienta de nuevo cuño, concebida además desde el principio conforme a la nueva cultura de los llamados "nativos digitales", con la que España aportará su colaboración en el buen desarrollo y el éxito final del ambicioso nuevo programa curricular que abre en la República Popular China la posibilidad de que millones de escolares preuniversitarios accedan al estudio del español. Este programa sin duda provocará a la vez un considerable aumento de los estudiantes universitarios de Filología española en China, para los que nacerán nuevas ofertas de trabajo, así como también para maestros de lengua procedentes de los países hispanohablantes, lo que apunta a uno de los parámetros que los investigadores del valor económico del español ya mencionados tuvieron en cuenta.

43 Pero a este valor hay que añadir otros como el primero que estamos contemplando: la globalidad de una lengua ecuménica, que satisface las necesidades de los países que la hablan como materna, pero que crece también en los demás porque como lengua extranjera proporciona beneficios que así son estimados por la población que se decide a estudiarla. Y añado ahora otro valor doblemente apreciable en el contexto de la globalización: el de la unidad.

En la historia de nuestra lengua común es obligado considerar tres momentos trascendentales. El primero es, obviamente, el fundacional, la constitución del romance castellano y su expansión por la Península ocupada por los árabes. El segundo comienza en 1492, el año de la Gramática de Nebrija, con la llegada de Colón a América. Y el tercero es el que hace del español una lengua ecuménica, la segunda por el número de hablantes nativos en todo el mundo: con este tercer momento me refiero al proceso de la independencia y constitución de las Repúblicas americanas a partir de finales del segundo decenio del Siglo XIX.

Momento crítico en el que ciertos augures vaticinaban un desarrollo semejante a lo que con la caída del Imperio Romano representó la fragmentación lingüística de la Romania. Y no fue así porque las nuevas Repúblicas soberanas, al tiempo que consolidaban el Estado, la nacionalidad, fijaban sus respectivos territorios y fronteras, organizaban la administración y abordaban el reto de la enseñanza de su ciudadanía creyeron útil el castellano o español como instrumento de cohesión, de integración 
nacional. De unidad. El español es la lengua que hoy es no por la Colonia, sino por la Independencia. Los sociolingüistas certifican que en 1820 hablaban español solo un veinte por ciento de los habitantes en la América hispana. Por lo que se refiere al castellano o español, los hispanohablantes, cada uno de los hispanohablantes, se siente hoy con toda legitimidad dueño de la lengua. Reside en ella como quien ocupa un lugar en el mundo. Sabe también que las palabras que la componen no solo sirven para decir, sino también para hacer; para crear, incluso, realidades.

En la unidad de nuestra lengua universal, bien perceptible hoy gracias a la fluida comunicación que la movilidad de las personas y la transmisión a través de los medios de nuestras respectivas hablas facilita, tuvo mucho que ver, en este trascendental siglo XIX, la labor académica. Unidad incomparable a la de cualquiera de las otras grandes lenguas. Unidad ortográfica plena. Unidad reconocida y justificada en la monumental Nueva gramática de la lengua española publicada en 2009, completada con la Fonética dos años después y elaborada desde el más escrupuloso planteamiento panhispánico. Unidad que no significa uniformidad, pues se enriquece con los distintos acentos, modismos y particularismos del español de todo el mundo.

Hace ahora 151 años, cinco decenios después de las independencias, la Real Academia Española, que ya había nombrado como miembro suyo correspondiente al gran maestro de nuestra lengua en el Siglo XIX, el venezolano/chileno Andrés Bello, elaboró un Reglamento para la fundación de Academias Americanas correspondientes, aprobado por la Junta de 24 de noviembre de 1870 a propuesta del Director, el Marqués de Molíns y de otros académicos.

El sucinto reglamento de 11 artículos viene precedido de una exposición de motivos que parece escrita desde un profundo sentimiento de fraternidad y exigencia de unidad, como bien se percibe en esta frase: «Los lazos políticos se han roto para siempre; de la tradición histórica misma puede en rigor prescindirse; ha cabido, por desdicha, la hostilidad, hasta el odio entre España y la América que fue española; pero una misma lengua hablamos, de la cual, si en tiempos aciagos que ya pasaron usamos hasta para maldecirnos, hoy hemos de emplearla para nuestra común inteligencia, aprovechamiento y recreo».

Y como fruto de este espíritu, se creó en 1871 la Academia colombiana de la lengua española, la decana, detrás de la RAE, de las hoy existentes. Muy pronto celebrará, pues, su sesquicentenario.

Encuentro en este texto fundamental el germen de la inspiración panhispánica que hoy felizmente rige la actividad de todas nuestras Academias. Fue en 1950 cuando el entonces presidente mexicano Miguel Alemán Valdés promovió la iniciativa de un primer "congreso de Academias de habla española". Las sesiones se celebraron en abril de 1951 y dieron origen a la Asociación de Academias de la Lengua Española (ASALE).

51 Pero ya en 1870 se hablaba, por ejemplo, de la necesidad de "activas y regulares comunicaciones". Pero, sobre todo, se afirmaba que «la Academia Española ha reconocido y proclamado que, sin el concurso de los españoles de América [lógicamente hay que entender por tales a los americanos que hablan español], no podrá formar el grande y verdadero Diccionario Nacional de la Lengua. Para ello convoca a sus hermanos, nacidos y puestos al otro lado de los mares...» Se llega a formular, en la misma línea, el desiderátum de una futura organización como ASALE, que llegará por parte de las Academias «formando entre todas una federación natural que no reconozca límites ni barreras dondequiera que sea lengua patria la lengua de Cervantes, cuyos 
pueblos (...) podrán formar diversas naciones, pero nunca perderán esta robusta y poderosa unidad, nunca dejarán de ser hermanos».

A la Academia decana, la colombiana, seguirán la ecuatoriana, la mexicana y otras más constituidas tanto en el siglo XIX como en el XX. Están todas las de los países americanos, incluidas la de Puerto Rico y la Academia Norteamericana de la Lengua Española, que acaba de cumplir 45 desde su creación. De 1924 data la Academia filipina, y la penúltima establecida hasta el momento, ya en pleno siglo XXI, ha sido la del único país de África que tiene el español como lengua oficial: la Academia Ecuatoguineana. Esa nómina puede darse por cerrada con una vigesimocuarta Academia, que no es otra que la del judeoespañol, la lengua que los judíos sefardíes, expulsados de España en 1492, mantuvieron viva hasta hoy en sus comunidades extendidas por gran parte de Europa, por el Imperio Otomano y algunos enclaves del Nuevo Mundo. La Academia Nacional del judeoespañol o ladino, iniciativa que ha sido aceptada por el gobierno de Israel, figura ya como correspondiente de la RAE por acuerdo del pleno de esta tomado por unanimidad el jueves 3 de octubre de 2019.

La declaración de 1870 por parte de la Real Academia Española que sentó el punto de partida de lo que hoy ya son las 24 corporaciones de ASALE habla de emplear desde ya el español «para nuestra común inteligencia, aprovechamiento y recreo». Ello nos lleva inevitablemente a otra de las dimensiones de la primera acepción de valor en el Diccionario académico: la de "proporcionar bienestar o deleite». Y esto es lo que la lengua aporta cuando se convierte en expresión artística, a través, fundamentalmente, de la literatura, pero también de la música y de las artes o expresiones escénicas, tanto en el registro culto como en popular.

Pocas literaturas como la que el Premio Miguel de Cervantes viene alentando desde 1976 pueden ofrecer un sistema tan integrado y estable de relaciones entre factores como tradición y renovación, escritores y público, centros y periferias, modelos y excepciones, creadores y mediadores, censura y libertad, críticos y lectores. Hablar, pues, de una literatura en dos continentes, o mejor, en cuatro continentes, tiene pleno sentido, más allá de lo meramente geográfico. La prueba está en los datos, y éstos se podrían multiplicar no sólo a partir de las personalidades y trayectorias de los escritores ya premiados, sino también de los que todavía no lo han sido.

El sistema literario correspondiente a nuestra lengua cuenta, efectivamente, con autores y lectores procedentes de cuatro continentes: Europa, América, Asia y África. En tres de ellos, Europa, Asia y África, es solo una nación la que los aporta: España, Filipinas y Guinea Ecuatorial, respectivamente. Pero en el importante capítulo de la mediación entre autores y lectores, el sistema debe intensificar el carácter transversal de la acción representada por los agentes y las casas editoriales, por la industria cultural, por la crítica y la información periodística, así como por la investigación históricoliteraria. Esa transversalidad funciona desde antiguo en lo que se refiere a las relaciones entre Europa y América, pero debe incrementarse considerablemente, sobre lo ya existente, en el caso de Guinea Ecuatorial y de Filipinas. Existe, sin embargo, una diferencia fundamental a este respecto: la emergencia de una renovada actividad creativa en español por parte de los poetas, narradores y autores teatrales ecuatoguineanos.

Que la literatura en lengua española fue, desde muy pronto, un factor de comunicación integradora entre los pueblos de ambos lados del Atlántico es un hecho de la más 
absoluta evidencia, como también lo es que siga constituyendo, junto a la propia lengua, el vínculo patrimonial más firme que nos une más allá de los avatares de la historia, incluidas las exigencias afirmadoras de las nacionalidades: como recuerda Carlos Fuentes, sin la lengua de la colonia no hubiese habido lengua de la independencia. Los escritores cubanos, argentinos, uruguayos, mexicanos, españoles, peruanos, colombianos, nicaragüenses y paraguayos que hasta el momento han merecido el Cervantes comparten un mismo idioma, que adquiere en sus plumas diferentes acentos literarios sumamente enriquecedores del acervo hispánico, pero participan a la vez, y desde diferentes perspectivas, de un sistema común en cuyo marco se institucionaliza una literatura.

Así como no es imaginable una literatura sin lengua, la mera presencia de ésta no es condición suficiente para la existencia de aquélla. Resultaría, no obstante, difícil cuestionar la certeza de que en un principio fue la palabra, pero también fue la denodada lucha del escritor, de cada escritor, por hacerse con las más expresivas para transmitir su percepción del mundo y abrirnos su yo, y por encontrar el orden mejor con que combinarlas.

60 Mas si la lengua en sí misma es ya un fenómeno de sociedad, sobre el que el genio individual del hablante sólo puede influir dentro de unos márgenes que los grandes artistas han sido capaces de ampliar extraordinariamente, otro tanto cabe decir de la literatura, que hoy por hoy se tiende a ver como un sistema complejo en el que, además del creador de los textos y de sus lectores, desempeñan un papel decisivo otros elementos que median entre unos y otros, contribuyendo a que los libros lleguen a sus destinatarios y adquieran toda la resonancia que instituciones como la crítica y la enseñanza, $u$ otras formas de recreación pueden proporcionarles. Entendidas así las cosas, cada factor, proceso o agente de este sistema literario participa y depende de todos los demás, y tan sólo desde esta perspectiva de conjunto se puede aprehender en un sentido pleno el significado de la literatura.

61 Bien destacó todo esto Octavio Paz en su discurso de Alcalá con motivo de la recepción del Premio Cervantes correspondiente a 1981 cuando afirmaba que por literatura no entendía simplemente una colección de autores y libros sino «una sociedad de obras» en la que el papel cocreador de los lectores es fundamental. Pero no lo es menos la presencia de una tradición literaria consolidada, con su abanico de temas, procedimientos y estilos, con su canon de autoridades comúnmente aceptadas, de una crítica y una didáctica atentas a su análisis e interpretación, y de una industria cultural que mediante las casas editoras, las revistas y suplementos literarios y otras empresas afines provea de cauce y soporte a la pura creación, y satisfaga las demandas de los lectores.

62 La literatura no termina con el momento misterioso y germinal de la escritura en solitario del poema, la novela, el ensayo o el drama, pues entonces el texto apenas si tiene vida propia más allá de su creador, de cuyo talento, percepción estética de la realidad, cosmovisión y demonios personales la obra viene a ser cabal reflejo. En cierto modo, es entonces cuando la obra literaria comienza a existir, y se abre para ella un vasto panorama en el que no caben limitaciones espaciales ni cronológicas. Palabra esencial en el tiempo era la poesía para Antonio Machado, y según T. S. Eliot, el conjunto de la literatura universal, y dentro de ella el conjunto de la literatura de cada país, tiene una existencia simultánea y compone un orden simultáneo. 
63 sí, y en cierto modo también compatriotas, es fácilmente asimilable a la vieja afirmación de que la patria de un escritor es la lengua, una y otra vez invocada por los ganadores del premio Cervantes: por ejemplo, así lo fue en 1983 por Luis Rosales y en 1992 por Francisco Ayala. Para Jorge Luis Borges, por su parte, la lengua es nuestra tradición, y tan sólo le es dado a cada escritor el modificarla levemente, idea que también agradaría a Eliot, pues para él, en contra de los excesos románticos, la originalidad de un autor brilla tanto más cuanto mejor se incardina en la tradición a la que pertenece. Por supuesto que ésta no comprende sólo la literatura escrita en una lengua, pero cuando ésta es tan amplia y fecunda como la española, constituye en sí misma todo un universo inagotable de recursos y posibilidades, tanto para los autores como para sus lectores. formulación eminente en una memorable intervención del premio Cervantes Sergio Ramírez en el Congreso Internacional de la Lengua Española que tuvo lugar en Cartagena de Indias en 2007.

El nicaragüense plantea desde el comienzo la angustia de «sentirse escritor de una lengua con el país por cárcel, oprimida dentro de las rejas de la comunicación y la expresión local». Menciona, así, a dos literatos víctimas de esa especie de claustrofobia lingüística, checo el uno y húngaro el otro, y recuerda también que «Nicaragua es un país más pequeño que la Hungría de Sandor Marai, o de lo que fue la antigua Checoslovaquia de Milán Kundera [en cuyo éxito internacional tuvieron que ver mucho sus traducciones al francés, añado por mi cuenta], y por eso me intriga, y me aterra, esa posibilidad de que nadie pudiera oírme más allá de mis fronteras, o la de quedarme alguna vez sin lengua». Y concluye con un párrafo insuperable con el que yo también quisiera, tomando de prestado su voz, quintaesenciar uno de los valores inmarcesibles del español, la lengua que satisface las necesidades espirituales y proporciona bienestar y deleite a todos los muchos que la hablamos. Cito para cerrar mi intervención con palabras más eminentes que las mías al compatriota de Rubén Darío y autor de Margarita, está linda la mar:

Digo todo esto, como quien se consuela con la realidad al despertar de un mal sueño, porque con el español me ocurre todo lo contrario a los padecimientos de los escritores de lenguas encerradas. Soy escritor en una lengua vasta, cambiante y múltiple, sin fronteras ni compartimientos [...]. Puedo volar toda una noche, de Managua a Buenos Aires, y siempre me estarán oyendo, estaré oyendo el español porteño desde mi español centroamericano [...] ningún otro idioma dueño de un territorio tan vasto. Me oirán en la Patagonia, y en Ciudad Juárez, un continente de por medio, y en el Caribe de las Antillas Mayores, y en el arco del Golfo de México, y del otro lado del dilatado Atlántico también me oirán, y oiré, en tierras de Castilla, y en las de Extremadura, y en las de León, en las de Aragón. Nos oiremos, hablaremos. Sabremos de qué estamos hablando, porque en la lengua, somos idénticos, estamos ungidos por la misma gracia. 


\section{BIBLIOGRAFÍA}

José Luis GARCíA DELGADO, José Antonio ALONSO, Juan Carlos JIMÉNEZ, Economía del español. Una introducción, Madrid, Ariel/Telefónica, 2008.

Ángel MARTín MUNICIO (ed.), El valor económico de la lengua española, Madrid, Espasa, 2003.

José María MERINO y Álex GRIJELMo [compiladores], Más de 555 millones podemos leer este libro sin traducción, Madrid, Taurus, 2019.

Guillermo Rojo y Mercedes SÁNCHEZ, El español en la red, Madrid, Ariel/Fundación Telefónica, 2010.

\section{RESÚMENES}

Cet article résume les recherches qui ont été menées sur la valeur économique de l'espagnol, qui le place à environ $16 \%$ du produit intérieur brut de l'Espagne selon une méthodologie d'analyse macroéconomique qui pourrait être appliquée à d'autres pays hispanophones. De même, les données démographiques qui en font la langue seconde par nombre de locuteurs natifs sont analysées. Les situations de l'espagnol aux États-Unis et en Chine sont spécifiquement étudiées. Aux USA, les hispanophones constituent déjà la première minorité, représentant $18 \%$ de la population, avec le passage, face à l'anglais, d'une situation de diglossie à une autre de bilinguisme. En Chine, depuis 2018, l'offre d'espagnol est décrétée dans le système éducatif, avec une augmentation considérable des études hispaniques à l'Université. Enfin, une autre dimension de la valeur de l'espagnol se démarque : l'unité qu'il maintient dans les quatre continents où il est parlé, et la richesse de la culture qui s'exprime en espagnol, ainsi que le potentiel de son système littéraire.

This article summarizes the research that has been carried out on the economic value of Spanish, which places it at around $16 \%$ of the Gross Domestic Product of Spain according to a macroeconomic analysis methodology that could be applied to other Spanish-speaking countries. Likewise, the demographic data that make it the second language by number of native speakers are analyzed. The situations of Spanish in the United States and in China are specifically studied. In USA, Spanish-speakers already constitute the first minority, with $18 \%$ of the citizenship, with the progress, in the face of English, from a situation of diglossia to another of bilingualism. In China, since 2018 the offer of Spanish has been decreed in the educational system, with a considerable increase in Hispanic studies at the University. Finally, another dimension of the value of Spanish stands out: the unity it maintains in the four continents in which it is spoken, and the richness of the culture that is expressed in Spanish, as well as the potential of its literary system.

ÍNDICE

Keywords: economy, culture, unit, demography, increase

Mots-clés: économie, culture, unité, démographie, augmenter 
AUTOR

DARÍO VILLANUEVA

Universidad de Santiago de Compostela

Real Academia Española 